\title{
Capital psicológico como mediador entre adicción al trabajo y burnout en trabajadoras en la industria de maquila en Guanajuato
}

\section{Psychological capital as mediator among work addiction and burnout in women workers in the maquila industry in Guanajuato}

\author{
Carlos Gabriel Colín Flores ${ }^{1}$
}

\begin{abstract}
Resumen
El objetivo de este estudio está orientado hacia el entendimiento de las relaciones que existen entre la adicción al trabajo, el capital psicológico y el burnout en una muestra de trabajadoras mexicanas en la industria de maquila en Guanajuato, en el que se buscó identificar si el capital psicológico, media entre las otras dos variables. Para este propósito, se calcularon las correlaciones de Spearman entre las variables de estudio y posteriormente se efectuó un análisis de mediación teniendo como variable independiente a la adicción al trabajo, variable mediadora el capital psicológico y variable dependiente el constructo burnout. Para el estudio se integró una muestra de 451 de trabajadoras de la industria maquiladora en Guanajuato, México. Los hallazgos del estudio indican una correlación fuerte y positiva $(r=+0.73)$ entre la adicción al trabajo y el burnout; así como correlaciones fuertes y negativas entre la adicción al trabajo y el capital psicológico $(r=-0.67)$, por una parte, y entre el capital psicológico y el burnout $(r=-0.62)$ por otra.

En cuanto al análisis de mediación, se pudo corroborar que el capital psicológico, media parcialmente entre la adicción al trabajo y cada dimensión del constructo burnout.
\end{abstract}

Palabras clave: capital psicológico, adicción al trabajo, desgaste por trabajo.

\begin{abstract}
The purpose of this study is to understand the relationship among work addiction, psychological capital and burnout, in a sample of woman mexican workers in the maquila industry in Guanajuato, identifying whether psychological capital, mediates the relationship between the other two variables. For this research, Spearman correlations between pairs of the studied variables were calculated. Subsequently, a mediation analysis was conducted, having work addiction as the independent variable, psychological capital as mediating variable, and burnout as dependent variable. A sample of 451 women workers in the maquila industry was integrated, in Guanajuato, México. The study findings show a strong and positive correlation between work addiction and burnout $(r=+0.73)$, as well as strong a inverse correlation between work addiction and psychological capital and burnout $(r=-0.67)$, on one hand, and between psychological capital and burnout $(\mathrm{r}=-0.62)$, on the other hand. Regarding the mediation analysis, the mediation of psychological capital between work addiction and burnout was partially confirmed.
\end{abstract}

Key words: psychological capital, work addiction, burnout.

Códigos JEL: I12, J28, J81

Artículo Recibido: 18 de Mayo de 2020

Artículo Aceptado: 13 de Junio de 2020

${ }^{1}$ Doctor en administración. Profesor-investigador de la Facultad de Economía y Negocios de la Universidad Anáhuac México, Campus Norte. E-mail: carlos.colinf08@anahuac.mx; carlos.colin01@gmail.com. 


\section{Introducción}

En un entorno social y de negocios cada vez más dinámico, competitivo y en constante cambio, el mundo del trabajo se ha modificado de manera profunda. Por ello, se ha generado una fuerza laboral más dispuesta a invertir su energía para mantenerse activa y presente laboralmente. Por ejemplo, las áreas de capital humano en muchas empresas ofrecen, como alternativas de apoyo al trabajo, tecnología portable para laborar, horarios flexibles y la modalidad de trabajo a distancia (Molino, Bakker y Ghislieri, 2016).

Estos cambios han contribuido a que los empleados tengan mayor dificultad para desconectarse del trabajo (Huyghebaert, Fouquereau, Lahiani, Beltou, Gimenes y Gillet, 2016), y a que la frontera entre los ambientes casa-trabajo-vida personal, se pierda, lo que los lleva a destinarle más tiempo a trabajar que a las actividades personales y familiares (Colín, 2019; Moyer, Aziz y Wuensch, 2017).

Los efectos de la sobrecarga de trabajo se han manifestado en los colaboradores (Salanova, LópezGonzález, Llorens, del Líbano, Vicente-Herrero y Tomás-Salvá, 2016), quienes, como consecuencia, han tenido un desbalance en su salud física, incrementado el conflicto trabajo-familia y provocando como resultado, desgaste por exceso de trabajo, también conocido como "burnout" (Clark, Michel, Zhdanova, Pui y Baltes, 2016; Schaufeli, Bakker, Van der Heijden y Prins, 2009).

Varios estudios indican que los adictos al trabajo son propensos a caer en este desbalance y a ser víctimas del síndrome de burnout (Colín, 2019; Colín y Simón, 2014; Schaufeli et al., 2009).

En este sentido, los colaboradores que exhiben tendencias adictivas al trabajo pueden percibir que el trabajo en casa y la conectividad de la que actualmente se goza -debido al acceso a internet y telefonía celular- pueden darles beneficios para seguir con su patrón de alta inversión de tiempo a actividades laborales y estar conectados permanentemente a ellas (Schaufeli et al., 2009).

Sin embargo, la investigación realizada sobre este tema muestra lo contrario, la alta cantidad de tiempo dedicada al trabajo, sin duda alguna les acarrea a los trabajadores consecuencias que deterioran su bienestar físico y psicológico, además de que tiene un impacto negativo en el desempeño de las organizaciones, las relaciones personales y familiares (Andreassen, 2014; Caruso, 2006; Colín, 2019; Colín y Simón, 2014; Clark et al., 2016).

Con base en los argumentos anteriores, resulta imperativo que se realicen estudios de investigación que lleven al entendimiento de las consecuencias de una alta inversión de tiempo laboral en los empleados y en las organizaciones, así como para comprender el proceso por el cual aparecen éstas.

El capital psicológico es la aplicación de las fortalezas del colaborador como recursos para afrontar las demandas laborales a las que se ve sometido (Luthans y Youssef, 2004; Seligman, 1998). Éste puede considerarse como un recurso positivo que pueden utilizar los trabajadores con comportamientos adictivos al trabajo para reducir los efectos indeseables, en particular el burnout (Luthans y Youssef, 2004).

Hay tres estudios -uno realizado en México, otro en China y uno más hecho en los Estados Unidos de América- que tratan sobre el efecto protector del capital psicológico, como recurso positivo, sobre el burnout en colaboradores con tendencias adictivas al trabajo (Colín, 2019; Moyer et al., 2017; Y. Wang, Liu, J. Wang, y L. Wang, 2012), por lo que resulta relevante profundizar en investigaciones sobre este aspecto, sobre todo para el entorno de negocios mexicano.

El objetivo de este estudio está orientado hacia el entendimiento de las relaciones que existen entre la adicción al trabajo, el capital psicológico y el burnout, además de identificar si el capital psicológico puede mediar la relación entre la adicción al trabajo y el burnout. En una muestra de trabajadoras de la industria maquiladora que se ubica en el estado de Guanajuato en México. 
Capital psicológico como mediador entre adicción al trabajo y burnout en trabajadoras en la industria de maquila en Guanajuato

\section{Marco teórico}

\section{Adicción al trabajo}

La adicción al trabajo ha sido definida por Oates (1971) como "una irresistible e incontrolable necesidad de trabajar de forma incesante que es tan fuerte que interfiere con una o más funciones de la vida de las personas" (p. 9).

Estudios realizados por Spence y Robbins (1992) han planteado que la adicción al trabajo es un síndrome, "en el cual, los adictos al trabajo demuestran un alto nivel de involucramiento en el trabajo, un impulso a trabajar potenciado por presiones internas en el individuo y bajo nivel de disfrute en el trabajo" (p. 162). Estas tres facetas estructuran los componentes comportamental, cognitivo y afectivo del síndrome que enmascara, al decir de Spence y Robbins, la triada del adicto al trabajo y sus consecuencias negativas para la persona.

Los adictos al trabajo no solamente trabajan muchas horas en horarios extensos, sino que también lo hacen arduamente, más allá de lo que puede esperarse de un colaborador promedio y esta indulgencia excesiva en la actividad laboral es, a menudo, a expensas de otras actividades de la persona (Clark et al., 2016; Colín, 2019; Colín y Simón, 2014; Schaufeli et al., 2009).

De acuerdo con varios autores, los adictos al trabajo, de forma sorprendente, no están motivados por el aspecto financiero o los requerimientos de la actividad laboral, sino más bien por una presión interna que le transmite al colaborador, a manera de una obsesión, la necesidad de estar trabajando y no hacerlo le produce una sensación de culpa o ansiedad (Clark et al., 2016; Colín y Simón, 2014; Schaufeli et al., 2009; Spence y Robbins, 1992).

Esta teoría de adicción al trabajo propone que el logro del placer en las personas adictas al trabajo deriva de su obsesión relacionada a la actividad laboral por sobre todas las cosas, por lo que es un constructo negativo para la persona, ya que le causa afectaciones, que se generan porque el individuo descuida los aspectos de su vida que no involucran al trabajo, y siente ansiedad cuando no está laborando, lo que causa una falta de disfrute de sus experiencias personales y a su vez una falta de satisfacción en su experiencia al trabajar, porque, para ella, la actividad laboral nunca es suficiente y nunca está lo bien que espera, pues siempre hay algo que mejorar -debido a su comportamiento perfeccionista-. Con ello, la salud física y psicológica de la persona adicta al trabajo puede verse afectada, sus recursos personales desgastarse por el estrés ocasionado por las largas jornadas de trabajo y sus relaciones personales, afectarse (Azziz y Zikcar, 2006; Clark et al., 2016; Ng, Sorensen y Feldman, 2007; Schaufeli et al., 2009; Van Beek, Taris y Schaufeli, 2011).

Por otra parte, se pueden manifestar tendencias adictivas al trabajo en los colaboradores, como respuesta a ambientes de trabajo caracterizados por altas demandas laborales, incluso si ellos no son necesariamente adictos al trabajo (Clark et al., 2016). Por ejemplo, hay varios indicadores relacionados con esta situación para los colaboradores, tales como laborar muchas horas o llevar trabajo a casa y la conexión o disponibilidad permanente con el uso de tecnología para el trabajo a distancia (Mazzetti, Schaufeli y Guglielmi, 2014).

Por lo que hay organizaciones que fomentan tendencias adictivas en los colaboradores mediante su cultura laboral que premia esta conexión laboral permanente con reconocimiento público a este tipo de comportamiento, promociones o premios económicos (Mazzetti et al., 2014)

Hay profesiones que tienen mayor riesgo de adicción al trabajo, tal y como lo indican Taris, Van Beek y Schaufeli (2012) en los resultados de su investigación, en la que encontraron para un estudio realizado en Europa que hay actividades profesionales que pueden desarrollar con mayor facilidad adicción al trabajo como son: profesores, contadores, consultores, gerentes, médicos y enfermeras. 


\section{Capital psicológico}

Luthans y Youssef (2004) introdujeron el concepto de comportamiento organizacional positivo que "consiste en aplicar positivamente los recursos humanos, orientando a éstos hacia el empleo de su capacidad psicológica y el potenciamiento de sus fortalezas que pueden ser medidas, desarrolladas y gestionadas para mejorar el desempeño de los colaboradores en el lugar de trabajo" (p. 164).

Como parte de sus investigaciones sobre el comportamiento organizacional positivo, Luthans y Youssef (2004) definieron al capital psicológico "como la aplicación de la capacidad psicológica positiva y de las fortalezas del colaborador como recursos para afrontar las demandas laborales a las que se ve sometido y que éste se encuentra integrado por cuatro dimensiones: la autoeficacia, la esperanza, el optimismo y la resiliencia" (p. 165).

El primer elemento del capital psicológico, la autoeficacia, se refiere a la confianza que tiene una persona en su habilidad para ser exitoso, mediante el uso de la automotivación, los recursos cognitivos y la ejecución de cursos de acción (Luthans y Youseff, 2004).

Por otra parte, la esperanza ha sido definida por Snyder, Sympson, Ybasco, Borders, Babyak y Higgins (1996) como "un conjunto de aspectos cognitivos que comprenden la voluntad de éxito determinación personal enfocada hacia el cumplimiento de metas-, proyección y redireccionamiento de metas -planes establecidos para conseguir metas-" (p. 323). Este concepto lo incorporan Luthans y Youseff (2004) como parte del constructo de capital psicológico.

El optimismo está basado en el trabajo de Seligman (1998) y refleja las expectativas de manejar resultados positivos (Luthans y Youseff, 2004).

Finalmente, la resiliencia "se refiere a la habilidad para superar el conflicto, la incertidumbre, adversidad o falla y refleja la capacidad de adaptarse a las demandas estresantes de la vida y salir adelante" (Luthans y Youseff, 2004) (p. 165).

Se ha descubierto que el capital psicológico está correlacionado positivamente con el soporte social, así como con el desempeño y el engagement laborales (Newman, Ucbasaran, Zhu y Hirst, 2014).

Los individuos con altos niveles de capital psicológico tienen expectativas positivas, confían en sus habilidades, incrementan sus esfuerzos y alcanzan altos niveles de satisfacción laboral (Avey, Reichard, Luthans y Mhatre, 2011; Newman et al., 2014).

Pfeffer (1998) indica en su libro The Human Equation, que ha encontrado en sus investigaciones en compañías como Southwest, General Electric, Microsoft y otras muchas más de tamaño grande, mediano y pequeño, que el capital psicológico se relaciona de forma inversa con constructos con connotaciones negativas como son el estrés laboral, sentimientos de inequidad, incertidumbre con el empleo y conflicto trabajo-familia, lo que sugiere que pudiera tener un carácter de recurso protector ante estos elementos.

\section{Burnout}

Muchos investigadores conceptualizan el fenómeno de burnout como un síndrome psicológico (Maslach, Schaufeli y Leiter, 2001) y es frecuentemente considerado como un resultado del estrés cotidiano que encara una persona después de algún tiempo de realizar su actividad laboral (Shirom, 2011).

La exposición de un colaborador al estrés cotidiano en el trabajo trae como resultado un deterioro gradual de los recursos de energía intrínseca de un individuo y las consecuencias de éste incluyen un desgaste emocional, fatiga física y debilitamiento cognitivo (Shirom, 2011). 
Capital psicológico como mediador entre adicción al trabajo y burnout en trabajadoras en la industria de maquila en Guanajuato

Maslach y colaboradores (2001) indican que, considerando el alto nivel de involucramiento y el estrés asociado que implica la adicción al trabajo, no sorprende que ésta se encuentre relacionada con el burnout. Además, identifican tres dimensiones clave que integran este constructo: el desgaste emocional, despersonalización y reducción de la eficacia personal.

Para ellos, el desgaste emocional es el componente de estrés en el síndrome y representa los sentimientos de agotamiento de los recursos físicos y emocionales del individuo. La despersonalización es el componente interpersonal del síndrome y refleja una respuesta negativa o excesivamente distante del individuo sobre varios aspectos del trabajo - otros empleados, colaboradores, clientes-. Finalmente, la reducción de la eficacia personal corresponde a la dimensión de la autoevaluación y se refiere a los sentimientos de incompetencia sobre la actividad laboral que se presentan con el síndrome.

A la vez, Maslach y colaboradores (2001) señalan que el síndrome de burnout se ha vinculado con algunos aspectos negativos como son: el incremento de ausencias por enfermedad -con certificación médica-, comportamientos organizacionales anormales o renuncias al trabajo (abandono). Los empleados víctimas del burnout que no dejan su organización y permanecen en ella aportan a la empresa una baja productividad, baja efectividad en su trabajo y baja satisfacción laboral (Landsbergis, Schnall, Belkic, Baker, Schwartz y Pickering, 2011; Shirom, 2011).

\section{Adicción al trabajo, capital psicológico y burnout}

La relación entre la adicción al trabajo y capital psicológico ha sido poco explorada (Lanzo, Aziz y Wuensch, 2016) y de los cuatro factores individuales que comprenden el capital psicológico, solo la autoeficacia ha sido examinada individualmente en relación con la adicción al trabajo (Clark et al., 2016).

A pesar de la falta de investigación, los componentes del capital psicológico sugieren un patrón opuesto al que sigue la adicción al trabajo, por lo que puede pensarse en que hay una relación inversa entre el capital psicológico y la adicción al trabajo (Moyer et al., 2017).

Cheung, C. S. Tang y S. Tang (2011) encontraron que la adicción al trabajo tiene una fuerte relación positiva con el burnout y a su vez que los individuos con alto capital psicológico experimentan escasos síntomas de burnout, lo que apoya el pensamiento de Moyer y colaboradores (2017) sobre la relación inversa entre adicción al trabajo y capital psicológico.

Por su parte Colin (2019) encontró en un estudio realizado en la Ciudad de México, en un grupo de colaboradores con profesiones que presentan tendencias adictivas al trabajo, que el capital psicológico puede mediar parcialmente entre la adicción al trabajo y el burnout.

\section{Adicción al trabajo y burnout}

Las tendencias de adicción al trabajo de los colaboradores contribuyen de manera importante a que desarrollen el síndrome de burnout (Maslach, 1986).

El razonamiento que apoya a esta idea procede de la teoría de la conservación de recursos, que indica que un individuo busca tanto adquirir como mantener sus recursos -por ejemplo, objetos, energías, características personales y condiciones que faciliten alguna habilidad para conseguir las metas deseadas(Maslach, 1986; Shirom, 2011). En línea con lo anterior, cuando una persona pierde o experimenta alguna amenaza de perder sus recursos, esta condición le causa estrés y eventualmente este desencadena el burnout (Maslach, 1986; Shirom, 2011).

Los adictos al trabajo invierten una cantidad muy importante de sus recursos como son el tiempo y energía en sus labores, además, de que piensan compulsivamente en su trabajo, lo que reduce su habilidad 
para desconectarse de él generándoles estrés. De igual manera, el consumo de tiempo reduce su participación en actividades de recuperación, lo que les arroja muchas consecuencias negativas como el incremento del conflicto trabajo-familia y una baja satisfacción con su vida (Clark et al., 2016), agotando sus recursos, lo que, a su vez, termina por provocar una condición de burnout (Schaufeli, et al., 2009).

\section{Capital psicológico y burnout}

El capital psicológico como un recurso personal positivo, es una habilidad que beneficia al colaborador para el alcance de sus metas (Luthans, Avolio, Avey y Norman, 2007). Además de acuerdo con la teoría de conservación de recursos, un individuo con un alto capital psicológico debe estar mejor equipado para combatir las restricciones relacionadas con la pérdida de recursos (Maslach, 1986; Shirom, 2011).

De acuerdo con Hobfoll y Shirom (2000), los individuos con una gran cantidad de recursos personales pueden ser menos afectados por la pérdida de recursos y así quedar protegidos.

Las características protectoras de los componentes individuales del capital psicológico han sido planteadas en la literatura. Por ejemplo, Bandura (1997) indica que los individuos con altos niveles de autoeficacia muestran bajos niveles de burnout. Esto porque cuando se enfrentan con las demandas y el estrés laboral, los individuos equipados con autoeficacia pueden tener una sensación de confianza en sus competencias para aplicar sus recursos cognitivos y ejecutar las acciones que son necesarias para calmar la presión a la que se ven sujetos.

Por otra parte, las características individuales como ser optimista pueden dar un mejor equipamiento a los colaboradores para manejar el estrés o presión a la que pueden estar sometidos por sus actividades cotidianas, ya que estos trabajadores optimistas cuentan con una habilidad para abordar positivamente las situaciones experimentadas (Mensah y Amponsah-Tawiah, 2016). Hallazgos similares hacen eco en la investigación sobre la resiliencia (Avey, Luthans y Jensen, 2009).

Mensah y Amponsah-Tawiah (2016) examinaron cada componente individual de capital psicológico como factor mediador en la relación entre estrés ocupacional y bienestar psicológico, y los resultados confirmaron que cada uno de estos componentes individuales mediaban esta relación.

En cuanto al burnout, los resultados de estudios realizados a profesores a nivel de estudios profesionales en China encontraron que el capital psicológico se relacionaba negativamente con todas las dimensiones del burnouty, que los profesores con alto nivel de capital psicológico estaban mejor habilitados para lidiar con el estrés en el lugar de trabajo y eran menos proclives a experimentar burnout (Cheung, et al., 2011; Y. Wang, et al., 2012).

Moyer y colaboradores (2017) indican que es factible que el capital psicológico tenga una cualidad protectora sobre el burnout para los colaboradores que desarrollen este recurso, esto con base en las observaciones empíricas de Cheung y colaboradores (2011), Mensah y Amponsah-Tawiah (2016) y Y. Wang y colaboradores (2012), así como en lo indicado en la teoría de la conservación de recursos (Maslach, 1986; Shirom, 2011).

\section{Hipótesis}

En vista de que el capital psicológico es justamente un nuevo constructo, la investigación realizada en asociación con el burnout y la adicción al trabajo, es escaza, se ha encontrado solamente un estudio sobre el tema en México (Colín, 2019) y un par de investigaciones en los Estados Unidos de América y China (Cheung, et al., 2011; Lanzo et al., 2016; Moyer et al., 2017; Y. Wang et al., 2012). No obstante, estos constructos y sus componentes individuales han sido investigados de manera independiente y sus 
Capital psicológico como mediador entre adicción al trabajo y burnout en trabajadoras en la industria de maquila en Guanajuato

resultados son tema de interés para la gestión organizacional y del talento humano (Avey et al., 2009; Clark et al., 2016; Maslach, 1986; Shirom, 2011; Mensah y Amponsah-Tawiah, 2016; Moyer et al., 2017).

Así que, en este estudio, se plantean dos hipótesis. En la primera, se busca comprobar la relación inversa del capital psicológico con la adicción al trabajo y el burnout, que ha sido encontrada previamente en China, Estados Unidos de América y México. En este caso, para una muestra de colaboradoras mexicanas que trabajan en empresas de la industria maquiladora en Guanajuato, México. En la segunda, se pretende entender si el capital psicológico como recurso positivo puede mediar la relación entre la adicción al trabajo y el burnout para reducir sus consecuencias negativas. Por ello se plantea las siguientes hipótesis específicas:

H1a. La adicción al trabajo presenta una relación positiva y estadísticamente significativa con cada componente individual del burnout (desgaste emocional, despersonalización y reducción de la eficacia personal).

H1b: El capital psicológico muestra una relación inversa y estadísticamente significativa con cada síntoma individual del burnout (desgaste emocional, despersonalización y reducción de la eficacia personal).

H1c: La adicción al trabajo tiene una relación inversa y estadísticamente significativa con el capital psicológico.

H2a. La relación entre adicción al trabajo y desgaste emocional puede ser mediada por el capital psicológico.

H2b. La relación entre la adicción al trabajo y la despersonalización puede ser mediada por el capital psicológico.

H2c: la relación entre la adicción al trabajo y reducción de la eficacia personal puede ser mediada por el capital psicológico.

\section{Metodología}

Este estudio es del tipo no experimental, ex post facto, transeccional de carácter descriptivo y explicativo.

\section{Muestra}

La muestra de participantes fue seleccionada en el corredor de empresas maquiladoras ubicado en la carretera Celaya - San Miguel de Allende, en el estado de Guanajuato, México -cerca del poblado Juventino Rosas, en el estado de Guanajuato, México-.

La muestra fue por conveniencia, en la que se entrevistó a mujeres que trabajan en empresas maquiladoras y que tuvieran al menos un año en el empleo actual.

Participaron 451 trabajadoras de empresas maquiladoras en este estudio. La muestra estuvo integrada por: 451 mujeres. El rango de edad fue de 19 a 47 años -Media $(M)=34$.2; Desviación estándar $(\mathrm{DE})=15.1-$.

Con fines descriptivos y como marco de referencia para la muestra estudiada se indica el nivel de educación: posgrado, 13\%; profesional, 47\%; técnico-bachillerato, 31\% y otro, 9\%. Los años de experiencia laboral de los participantes, van de un año, hasta 23 años $-\mathrm{M}=2.2$; $\mathrm{DE}=1.7$-. Las horas trabajadas por semana van de 48 a 68 horas $-\mathrm{M}=52.9 ; \mathrm{DE}=4.8$. 


\section{Aplicación del cuestionario}

Se contrató a un grupo de encuestadores a quienes se les dio capacitación para aplicar el cuestionario al grupo objetivo de estudio mediante el uso de tabletas electrónicas. Los encuestadores, aplicaron el cuestionario a la salida de las empresas del corredor industrial ubicado en la carretera Celaya- San Miguel de Allende.

Se les pidió a los encuestadores que aplicaran los cuestionarios de datos generales, adicción al trabajo, burnout y capital psicológico. Al final se obtuvo 451 cuestionarios útiles, lo que dio una efectividad del $69.5 \%$ de la muestra.

\section{Instrumentos de medición}

\section{Adicción al trabajo}

La adicción al trabajo fue medida utilizando la versión de 10 ítems de la Dutch Work Addiction Scale (DUWAS) en español (Del Líbano, Llorens, Salanova y Schaufeli, 2010) y que fue validada para ejecutivos mexicanos por Colín y Simón (2014).

La escala DUWAS investiga el sentir de los participantes, con relación a su trabajo, y refleja dos componentes de adicción al trabajo: trabajar compulsivamente (TC) y trabajar excesivamente (TE). Un ejemplo de los ítems es el siguiente: "siento que hay algo en mi interior que me impulsa a trabajar incesantemente" (TC) y "generalmente estoy ocupado y tengo muchos asuntos entre manos" (TE). Las respuestas se dan en una escala de frecuencia subjetiva de cinco puntos que van de 1 "nunca o casi nunca" a 5 "siempre o casi siempre". Mayores puntuaciones muestran mayores niveles de adicción al trabajo.

La consistencia interna fue medida para este estudio con un coeficiente de alfa de Cronbach de 0.90 y el análisis factorial ejecutado mediante el método de componentes principales con rotación varimax, aportó dos factores: trabajar excesivamente y trabajar compulsivamente. Ambos explicaron el $77 \%$ de la varianza.

La prueba de factor único generó una varianza común del 12\% -si hubiera algún problema de varianza asociada al método, el factor debería explicar el $50 \%$ o más de la varianza como indican P. Podsakoff, Mackenzie, Lee, y N. Podsakoff (2003).

\section{Capital psicológico}

El capital psicológico fue medido empleando el cuestionario de Luthans et al. (2007) que consta de 24 ítems y mide las cuatro dimensiones del constructo, en su versión en español, validada por Azanza, Domínguez, Moriano y Molero (2014). En este estudio el interés fue medir el capital psicológico como constructo y no las dimensiones en particular, aunque para ello se aplicó el cuestionario como lo presentan los autores con sus cuatro dimensiones.

Se empleó una escala tipo Likert para captar las respuestas, que va de muy en desacuerdo "1" a muy de acuerdo " 6 ".

Para cada una de las cuatro dimensiones se evaluó seis ítems.

En el caso de la autoeficacia, se muestra un ejemplo de los ítems: "me siento seguro analizando un problema a largo plazo para encontrar una solución". Para la esperanza, un ejemplo de los ítems es: "hay muchas formas de darle la vuelta a cualquier problema". Para la dimensión de optimismo, un ejemplo de los ítems es: "en lo que respecta a mi trabajo, siempre veo el lado bueno de las cosas" y, finalmente para 
Capital psicológico como mediador entre adicción al trabajo y burnout en trabajadoras en la industria de maquila en Guanajuato

evaluar a la resiliencia, un ejemplo de los ítems es: "puedo superar las épocas laborales difíciles porque ya me he enfrentado antes a las dificultades".

La consistencia interna para el constructo del capital psicológico medido con el coeficiente alfa de Cronbach fue de 0.86 y el análisis factorial realizado mediante el método de componentes principales con rotación varimax, aportó cuatro factores: autoeficacia, esperanza, optimismo y resiliencia, los cuales explicaron el $81 \%$ de la varianza.

La prueba de factor único generó una varianza del 19\%, lo que parece indicar que no hay un problema de varianza asociado al método (P. Podsakoff et al., 2003).

\section{Burnout}

El burnout, se midió con la escala Maslach y Jackson (1981), en su versión en español y que consta de 22 ítems. Los entrevistados fueron cuestionados en relación con cada afirmación del cuestionario, a la cual debían contestar empleando una escala de frecuencia subjetiva que va de "0" nunca a "6" todos los días.

En esta escala, se evalúa tres dimensiones: desgaste emocional, despersonalización y reducción de la eficacia personal. A continuación, se muestra ejemplos de los ítems para cada factor. Desgaste emocional con nueve ítems, por ejemplo: "me siento emocionalmente agotado por mi trabajo". En el caso de despersonalización la escala está compuesta por cinco ítems, por ejemplo: "siento que me he hecho más duro al tratar a los colaboradores". La escala de la eficacia personal está integrada por ocho ítems, por ejemplo: "siento que puedo entender fácilmente a los colaboradores".

La consistencia interna para este estudio medida con el coeficiente alfa de Cronbach fue: burnout, 0.82; desgaste emocional, 0.85 ; despersonalización, 0.86 y reducción de la eficacia personal, 0.91 y el análisis factorial realizado mediante el método de componentes principales con rotación varimax, arrojó tres factores: desgaste personal, despersonalización y reducción de la eficacia personal. Los factores explican el $74 \%$ de la varianza.

La prueba de factor único mostró una varianza común del 17\%, lo que parece indicar que no hay un problema de varianza asociado al método (P. Podsakoff et al., 2003)

\section{Análisis de datos}

Se empleó el coeficiente de correlación de Spearman para examinar la relación entre las variables.

La hipótesis relativa a los efectos directos y la mediación de las variables fueron probadas mediante el proceso de análisis condicional basado en el análisis de regresión de mínimos cuadrados, empleando la técnica de bootstrapping -remuestreo-, un procedimiento de remuestreo no paramétrico que no asume normalidad y que involucra la extracción de varios miles de submuestras (10,000 en este caso) de la colección de datos. A través del bootsrapping, los efectos de la distribución son aproximados empíricamente y son empleados para calcular intervalos de confianza.

Los modelos analizados en este estudio corresponden al modelo conceptual de la plantilla número 4 de Hayes y para ello se empleó el software SPSS versión 20 y la macro PROCESS (Hayes, 2017).

\section{Análisis de resultados}

Se presentan los resultados de las correlaciones bivariadas de Spearman entre las variables de estudio y para clasificar la intensidad de la correlación se empleó la recomendación de los puntos de corte 
de Black (2011) - con base en el coeficiente de Spearman la correlación fuerte, con un coeficiente mayor a 0.6 ; moderada de 0.4 a 0.6 y baja, menor a $0.4-$.

La tabla 1 muestra las correlaciones bivariadas de Spearman entre las variables de estudio. En ella, se aprecia que la variable adicción al trabajo tiene una correlación fuerte y positiva con las variables burnout $\mathrm{r}=0.73, p<0.001$, IC al 95\% (0.68, 0.77); desgaste emocional $\mathrm{r}=0.78, p<0.001, \mathrm{IC}$ al $95 \%(0.74,0.82) \mathrm{y}$ una correlación fuerte y positiva con las variables despersonalización $r=0.69, p<0.001$, IC al 95\% (0.63, 0.74 ) y la variable reducción de la eficacia personal $r=0.58, p<0.001$, IC al $95 \%(0.54,0.63)$.

Por otro lado, la variable capital psicológico presenta una correlación fuerte y negativa con las variables desgaste emocional $\mathrm{r}=-0.67, p<0.001$, IC al 95\% $(-0.72,-0.64)$, despersonalización $\mathrm{r}=-0.64, p$ $<0.001$, IC al $95 \%(-0.69,-0.59)$ y moderada y negativa con la reducción de la eficacia personal $r=-0.56$, $p<0.001$, IC al 95\% (-0.60, -0.52).

En cuanto a la relación entre adicción al trabajo y capital psicológico, esta muestra una correlación fuerte y negativa $r=-0.65, p<0.001$, IC al 95\% $(-0.69,-0.59)$.

\section{Tabla 1}

Estadísticos descriptivos, correlaciones bivariadas de las variables estudiadas ( $N=451$ )

\begin{tabular}{|c|c|c|c|c|c|c|c|c|}
\hline \multicolumn{9}{|c|}{ Promedio Desviación } \\
\hline & $(\mathrm{M})$ & Estándar (DE) & 1 & 2 & 3 & 4 & 5 & 6 \\
\hline 1. Adicción al trabajo & 3.58 & 0.69 & 1 & & & & & \\
\hline 2. Capital Psicológico & 2.59 & 0.71 & $-0.65 * *$ & 1 & & & & \\
\hline 3. Burnout & 3.47 & 0.54 & $0.73^{* *}$ & $-0.62 * *$ & 1 & & & \\
\hline 4. Desgaste emocional & 3.35 & 0.55 & $0.78^{* *}$ & $-0.67^{* *}$ & $0.82 * *$ & 1 & & \\
\hline 5. Despersonalización & 3.58 & 0.53 & $0.69 * *$ & $-0.64 * *$ & $0.77^{* *}$ & $0.80 * *$ & 1 & \\
\hline 6. Reduccion de la eficacia persol & 3.62 & 0.54 & $0.58^{* *}$ & -0.56 ** & $0.71 * *$ & 0.61 ** & $0.57^{* *}$ & 1 \\
\hline
\end{tabular}

El análisis de mediación se realizó empleando la macro PROCESS en SPSS versión 20 y los resultados pueden apreciarse en las figuras 1,2 y 3. 
Capital psicológico como mediador entre adicción al trabajo y burnout en trabajadoras en la industria de maquila en Guanajuato

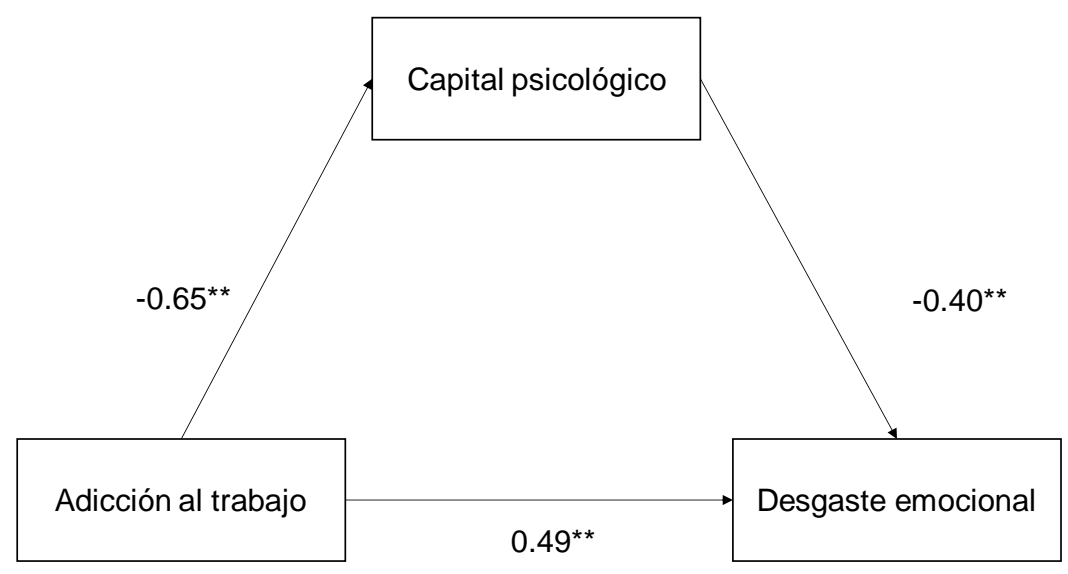

Nota: ${ }^{* *} p<0.001$

Fuente. (Elaboración propia).

Figura 1. Efecto mediador del capital psicológico sobre el desgaste emocional en presencia de adicción al trabajo.

El efecto total de la adicción al trabajo sobre el desgaste emocional $r=0.75$, se separó en el efecto directo $0.49, p<0.001$, IC al $95 \%(0.43,0.52)$ y el efecto indirecto $0.26, p<0.001, \mathrm{IC}$ al $95 \%(0.21,0.34)$. El $34.7 \%$ del efecto total corresponde al efecto indirecto.

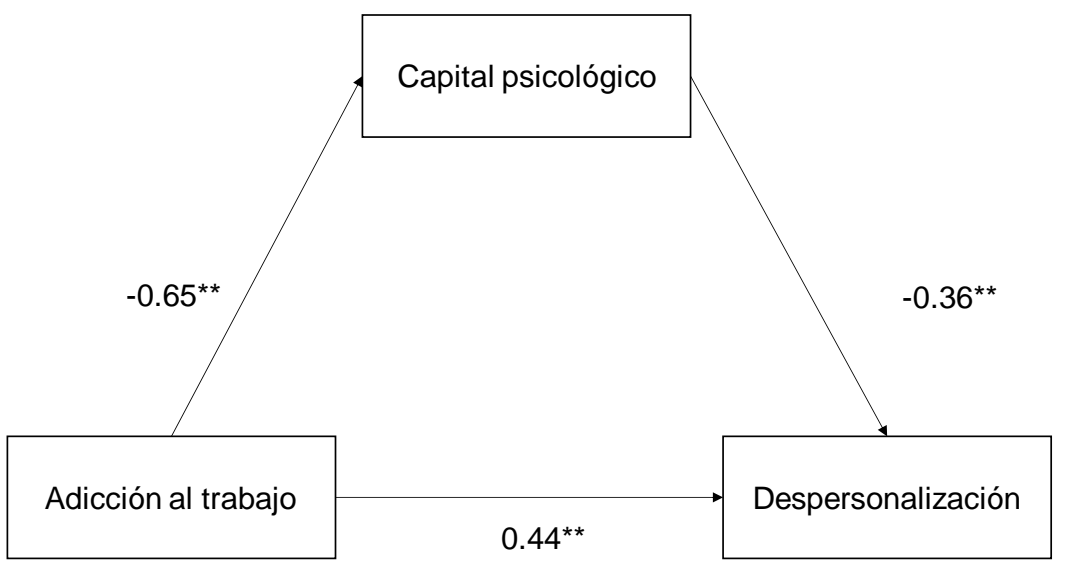

Nota: ${ }^{* *} p<0.001$

Fuente. (Elaboración propia).

Figura 2. Efecto mediador del capital psicológico sobre la despersonalización en presencia de adicción al trabajo. 
El efecto total de la adicción al trabajo sobre la despersonalización $r=0.67$, se separó en el efecto directo $0.44, p<0.001$, IC al $95 \%(0.39,0.49)$ y el efecto indirecto $0.23, p<0.001, \mathrm{IC}$ al $95 \%(0.17,0.29)$. El $34.3 \%$ del efecto total corresponde al efecto indirecto.

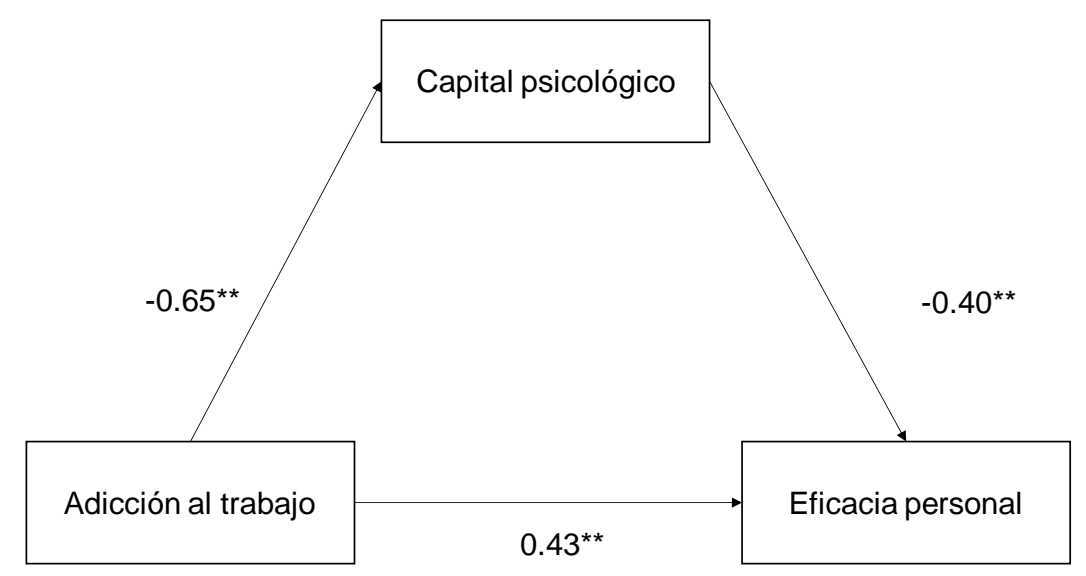

Nota: ${ }^{* *} p<0.001$

Fuente. (Elaboración propia).

Figura 3. Efecto mediador del capital psicológico sobre la eficacia personal en presencia de adicción al trabajo.

El efecto total de la adicción al trabajo sobre la reducción de la eficacia personal $r=0.69$, se separó en el efecto directo $0.43, p<0.001, \mathrm{IC}$ al $95 \%(0.38,0.47)$ y el efecto indirecto $0.26, p<0.001$, IC al $95 \%$ $(0.22,0.29)$. El $37.7 \%$ del efecto total corresponde al efecto indirecto.

Los resultados muestran una mediación parcial del capital psicológico sobre las dimensiones del burnout en empleados con características adictivas al trabajo.

\section{Conclusiones}

Como ha sido indicado en la introducción de este trabajo, en tiempos recientes los colaboradores en las empresas han dedicado más tiempo a trabajar como consecuencia de varios aspectos, entre ellos el incremento en la competencia por puestos de trabajo y la reducción de personal por optimización de costos en los procesos de negocio; la incorporación de la tecnología que extiende la presencia del empleado a lugares más allá de la oficina o sitio físico de trabajo y con ello la exigencia de contar de manera permanente con su disponibilidad (Schaufeli, et al., 2009; $\mathrm{Ng}$ et al., 2007).

La sobrecarga de trabajo ha traído Indudablemente efectos adversos que han sido documentados ampliamente en la literatura, y éstos incluyen aspectos como conductas adictivas al trabajo, síndrome de adicción al trabajo, síndrome burnout, afectaciones a la salud y conflicto trabajo-familia (Cheung, et al., 2011; Clark et al., 2011; 2016; Colín,2019; Maslach, 1986; Shirom, 2011).

Como estos efectos pueden afectar varios aspectos de la vida de los empleados, es conveniente que se realicen investigaciones para entender las consecuencias que tienen estas altas inversiones de tiempo en el trabajo y en particular las relaciones entre adicción al trabajo, capital psicológico, burnout y otras situaciones vinculadas con el estrés laboral (Clark et al., 2016; Colín, 2019; Ng et al., 2007). 
Capital psicológico como mediador entre adicción al trabajo y burnout en trabajadoras en la industria de maquila en Guanajuato

Varios autores han indicado que los recursos positivos del empleado como pueden ser el capital psicológico o el engagement laboral, entre otros, pueden ayudar a cumplir con las tareas actuales de los colaboradores y sobrellevar las largas jornadas de trabajo y estrés que ocasionan (Cheung et al., 2011; Lanzo et al., 2016; Luthans y Youssef, 2004; Mensah y Amponsah-Tawiah, 2016; Moyer et al., 2017; Seligman, 1998; Y. Wang et al., 2012).

No obstante, los recursos positivos con los que cuenta el empleado son susceptibles de desgaste a lo largo del tiempo, debido al estrés al que está sometido el colaborador y en el largo plazo existe la posibilidad que experimente consecuencias negativas como el burnout, por lo que resulta importante entender el manejo de aspectos como el capital psicológico o el engagement laboral para proteger al trabajador (Luthans et al., 2007).

La relación entre el capital psicológico y la adicción al trabajo no ha sido suficientemente estudiada. A pesar de ello, por hallazgos en estudios previos, se ha encontrado que el capital psicológico tiene una relación inversa con el burnout y éste último tiene a su vez una relación positiva con la adicción al trabajo, por lo que puede pensarse que el capital psicológico y la adicción al trabajo presentan una relación inversa (Cheung et al., 2011; Clark et al., 2016; Colín, 2019).

Al momento de realizar esta investigación, los únicos estudios encontrados en la literatura que exploran la relación entre la adicción al trabajo y el capital psicológico son los de Colín (2019), Lanzo et al. (2016) y Moyer et al. (2017), en los que se encontró una relación inversa entre estas dos variables.

En este estudio para colaboradoras mexicanas que trabajan en la industria maquiladora de Guanajuato, México, se obtuvo resultados similares a los encontrados en investigaciones previas.

Las colaboradoras que trabajan en la industria de maquila en Guanajuato que presentan tendencias adictivas al trabajo o con adicción al trabajo, mostraron una correlación positiva fuerte con el burnout y las variables que integran el constructo burnout y que son: el desgaste emocional, la despersonalización y la reducción en la eficacia personal, lo cual permite corroborar la $\mathrm{H} 1 \mathrm{a}$. Esto en la misma dirección que lo encontrado por Clark et al. (2016), Colín (2019) y por Schaufeli et al. (2009).

Adicionalmente, los resultados mostraron una correlación fuerte e inversa entre los constructos capital psicológico y burnout, además, con las variables que integran el constructo burnout -desgaste emocional, despersonalización y reducción en la eficacia personal-, lo que permite confirmar la H1b. En línea con lo encontrado en China por Cheung et al. (2011) y Y. Wang et al. (2012); en Ghana por Mensah y AmponsahTawiah (2016), en Estados Unidos de América por Moyer et al. (2017) y en México por Colín (2019).

En relación con la $\mathrm{H} 1 \mathrm{c}$, los resultados indicaron una relación fuerte e inversa entre los constructos adicción al trabajo y capital psicológico, por lo que se confirma la hipótesis, en concordancia con lo planteado por Cheung et al. (2011); Colín (2019) y Lanzo et al. (2016).

En cuanto al rol de mediación del capital psicológico entre el constructo de adicción al trabajo como variable independiente y las variables que integran el constructo burnout como variable dependiente desgaste emocional, despersonalización y reducción en la eficacia personal-, los resultados indican una mediación parcial del capital psicológico -el efecto indirecto es superior al 30\% del efecto total- entre la adicción al trabajo y las variables desgaste emocional, despersonalización y reducción de la eficacia personal -integrantes del constructo burnout -, lo que permite confirmar parcialmente H2a, H2b y H2c.

Esos resultados coinciden con lo encontrado por Colín (2019) y Moyer et al. (2017), en la que hay una mediación parcial de capital psicológico entre la adicción al trabajo y las variables desgaste emocional y despersonalización y reducción de la eficacia personal. En el caso de los estudios realizados por Colín (2019) y Moyer y colaboradores (2017), se encontró una mediación débil entre la adicción al trabajo y la reducción de la eficacia personal, que a su parecer puede explicarse por factores de premio recompensa 
a los colaboradores, en los que se premia en el corto plazo por seguir comportamientos adictivos -aumentos de sueldo, bonos o promociones- que no impactan a la eficacia personal.

No obstante, en el largo plazo, existe la posibilidad de que estos estímulos temporales acaben siendo parte de la condición operativa del trabajo y dejen de ser percibidos como extraordinarios, trayendo como consecuencia burnout, por lo que para poder confirmar o descartar esta condición se requiere de un estudio longitudinal que evalúe esta condición como sugieren Moyer y colaboradores (2017).

En la investigación realizada para las trabajadoras de la industria maquiladora de Guanajuato, la moderación del capital psicológico sobre las variables adicción al trabajo y reducción de la eficacia personal, no mostró una moderación débil, situación que pudiera estar asociada a que su rol como jefas de familia, les impulsa a ser eficaces para cumplir con sus responsabilidades en el hogar, esta situación deberá corroborarse con estudios más profundos sobre el tema.

Aun cuando este estudio aportó hallazgos importantes sobre la relación entre adicción al trabajo, capital psicológico y burnout, así como sobre el papel mediador del capital psicológico entre la adicción al trabajo y burnout para una muestra de trabajadoras de la industria maquiladora en Guanajuato, México, hay algunas limitaciones en él, que deberán de replantearse para abrir líneas futuras de investigación.

Los resultados provienen de un estudio transeccional por lo que esta condición limita las conclusiones que pueden hacerse de él y no permite hacer inferencias causales, por lo que resultaría muy interesante proseguir con la investigación con estudios longitudinales que permitan hacer generalizaciones sobre los hallazgos.

Por otra parte, la muestra del estudio está sesgada intencionalmente a mujeres trabajadoras de la industria maquiladora, por lo que podría ampliarse la muestra para buscar resultados de sectores poblacionales más típicos y amplios, además del sesgo que implicó un muestreo por conveniencia. Asimismo, la información fue recolectada por autoinforme de los participantes, lo que trae consigo otra fuente de sesgo potencial en sus respuestas.

\section{Implicaciones prácticas}

Trabajar en exceso trae consecuencias negativas para la persona -en su salud-, sus relaciones conflicto trabajo-familia- y también para la empresa -pérdida de compromiso y productividad ocasionada por el burnout-.

Como puede apreciarse de los resultados de este estudio para trabajadoras mexicanas y de otros estudios nacionales e internacionales (Cheung et al., 2011; Colín, 2019; Lanzo, et al., 2016; Moyer et al., 2017), la adicción al trabajo está fuertemente relacionada con el burnout.

Un recurso que puede emplearse por las empresas como mediador del efecto de los comportamientos adictivos al trabajo y sus consecuencias como el burnout, es el capital psicológico, ya que éste tiene un rol de mediación entre estas variables y puede ayudar a reducir los efectos del burnout, causados por trabajar obsesivamente y en exceso, como se indica en los resultados de esta investigación.

Por lo anterior, una aplicación práctica de los resultados de esta investigación puede ser la generación de programas que fomenten el desarrollo del capital psicológico de los colaboradores como parte de su formación institucional, para darles herramientas que fortalezcan su autoeficacia, optimismo, esperanza y resiliencia, variables que integran el constructo de capital psicológico. Este tipo de programas podrían denominarse cómo educación para el trabajo y bienestar laboral, y bien podrían registrarse en la Secretaría del Trabajo y Previsión social como elementos que aporten positivamente hacia el desarrollo y bienestar del personal. 
Capital psicológico como mediador entre adicción al trabajo y burnout en trabajadoras en la industria de maquila en Guanajuato

Además, puede ser recomendable generar programas de intervención en el caso de empleados con un burnout manifiesto para contenerlo y evitar el fomento de prácticas de trabajo en exceso que invadan otras actividades del trabajador y desbalanceen su equilibrio de vida (Lambert, Minor, Wells y Hogan, 2016; Weigl, Stab, Herms, Angerer, Hacker y Glaser, 2016).

A manera de resumen, puede decirse que los hallazgos de este estudio complementan la literatura existente.

En primer lugar, se confirma la relación entre las diferentes variables de estudio, en las que hay una correlación fuerte y positiva entre la adicción al trabajo y burnout. Por otra parte, hay una correlación moderada pero negativa entre el capital psicológico y burnout y, finalmente, una correlación moderada y negativa entre la adicción al trabajo y el capital psicológico.

En segundo lugar, se confirma que el capital psicológico puede mediar parcialmente entre los constructos de adicción al trabajo -variable independiente- y el desgaste por trabajo -variable dependiente, lo que da la oportunidad de desarrollar este recurso positivo en las empresas para que medie entre el estrés laboral ocasionado por las largas jornadas laborales y los comportamientos adictivos al trabajo de los colaboradores.

En tercer lugar, se identificó la posibilidad de realizar estudios longitudinales sobre estas variables para entender la relación causal entre ellas y a su vez comprender más ampliamente estos fenómenos para proponer alternativas de gestión que apoyen a los colaboradores y a la organización.

Finalmente, se presentó una propuesta para generar programas de desarrollo del capital psicológico en las organizaciones con el fin de proveer recursos positivos a los colaboradores.

\section{Referencias}

Andreassen, C. S. (2014). Workaholism: an overview and current status of the research. Journal of Behavioral Addictions, (1), 1-11. <a href="https://doi.org/10.1556/JBA.2.2013.017"target="_blank"> https://doi.org/10.1556/JBA.2.2013.017</a>.

Avey, J. B., Luthans, F., y Jensen, S. M. (2009). Psychological capital: a positive resource for combating employee stress and turnover. Human Resource Management, 48(5), 677-693. <a href="https://doi.org/10.1002/hrm.20294" target="_blank"> https://doi.org/10.1002/hrm.20294 </a>.

Avey, J. B., Reichard, R. J., Luthans, F., y Mhatre, K. H. (2011). Meta-analysis of the impact of positive psychological capital on employee attitudes, behaviors, and performance. Human Resource Development Quarterly, 22(2), 127-152. <a href="https://doi.org/10.1002/hrdq.20070" target="_blank">https://doi.org/10.1002/hrdq.20070</a>.

Azanza, G., Domínguez, A. J., Moriano, J. A., y Molero, F. J. (2014). Capital psicológico positivo. Validación del cuestionario PCQ en España. Anales de la Psicología, 39(1), 294-301. <a href="https://dxdoi.org/10.6018/anaesps.30.1.153631"target="_blank">https://dx-

doi.org/10.6018/anaesps.30.1.153631</a>

Balducci, C., Avanzi, L., Consiglio, C., Fraccaroli, F., y Schaufeli, W. A. (2015). Cross-National Study on the Psychometric Quality of the Italian Version of the DutchWork Addiction Scale (DUWAS). European Journal of Psychology Assessment, 33, 1-7. 
Bandura, A. (1997). Self-Efficacy: The Exercise of Control. New York, USA: Freeman.

Black, K. (2011). Estadística en los negocios. México: Grupo Editorial Patria.

Caruso, C. C. (2006). Possible broad impacts of long work hours. Industrial Health, 44(4), 531-536. <a href="https://doi.org/10.2486/indhealth.44.531" https://doi.org/10.2486/indhealth.44.531</a>.

target="_blank">

Cheung, F., Tang, C. S., y Tang, S. (2011). Psychological capital as a moderator between emotional labor, burnout, and job satisfaction among school-teachers in China. International Journal of Stress Management, 18(4), 348-371. <a href="https://doi.org/10.1037/a0025787" target="_blank"> https://doi.org/10.1037/a0025787 </a>.

Clark, M. A., Michel, J. S., Zhdanova, L., Pui, S. Y., y Baltes, B. B. (2016). All work and no play? A metaanalytic examination of the correlates and outcomes of workaholism. Journal of Management, 42(7), $1-38$.

Colín, C. G. (2019). El rol mediador del capital psicológico entre la adición al trabajo y el burnout en trabajadores mexicanos. The Anáhuac Journal, 19(2), 71-97. <a href="https://doi.org/10.36105/theanahuacjour.2019v19n2" target="_blank"> https://doi.org/10.36105/theanahuacjour.2019v19n2 </a>

Colín, C. G., y Simón. N. (2014). Adicción al trabajo, satisfacción y desempeño laboral en ejecutivos mexicanos. Psicología Iberoamericana, 22(2), 17-27.

Del Líbano, M., Llorens, S., Salanova, M., y Schaufeli, W. (2010). Validity of a brief workaholism scale. Psychotema, 22, 143-150.

Hayes, A.F. (2017). Introduction to mediation, moderation, and conditional process analysis: A regressionbased approach. London, GB: The Guilford Press.

Hobfoll, S. E., y Shirom. A. (2000). Conservation of resources theory: applications to stress and management in the workplace. In R. T. Golembieswski (Ed.), Handbook of Organization Behavior, (57-81). New York, USA: Dekker.

Huyghebaert, T., Fouquereau, E., Lahiani, F., Beltou, N., Gimenes, G., y Gillet, N. (2016), Examining the longitudinal effects of workload on ill-being through each dimension of workaholism. International Journal of Stress Management, 25(2), 144-162. <a href="https://doi.org/10.1037/str0000055" target="_blank"> https://doi.org/10.1037/str0000055 </a> .

Lambert, E. G., Minor, K. I., Wells, J. B., y Hogan, N. L. (2016). Social support's relationship to correctional staff job stress, job involvement, job satisfaction, and organizational commitment. The Social Science Journal, 53(1), 22-32. <a href="https://doi.org/10.1016/j.soscij.2015.10.001" target="_blank"> https://doi.org/10.1016/j.soscij.2015.10.001</a>.

Landsbergis, P. A., Schnall, P. L., Belkic, K. L., Baker, D., Schwartz, J. E., y Pickering, T. G. (2011). Workplace and cardiovascular disease: relevance and potential role for occupational health psychology. In J.C. Quick, L.E. Tetrick, (Eds.), Handbook of Occupational Health Psychology (243264). Washington, USA: American Psychological Association. 
Capital psicológico como mediador entre adicción al trabajo y burnout en trabajadoras en la industria de maquila en Guanajuato

Lanzo, L., Aziz, S., y Wuensch, K. L. (2016). Workaholism and incivility: stress and psychological capital's role. International Journal of Workplace Health Management, 9(2), 165-183.

Luthans, F., Avolio, B. J., Avey, J. B., y Norman, S. M. (2007). Positive psychological capital: measurement and relationship with performance and satisfaction. Personnel Psychology, 60(3), 541-572. <a href="https://doi.org/10.1111/j.1744-6570.2007.00083.x" target="_blank"> https://doi.org/10.1111/j.1744-6570.2007.00083.x </a> .

Luthans, F., y Youssef, C. M. (2004). Human, social, and now positive psychological capital management: investing in people for the competitive advantage. Organizational Dynamics, 33(2), 143-160. <a href= "https://doi.org/10.1016/j.orgdyn.2004.01.003" target="_blank"> https://doi.org/10.1016/j.orgdyn.2004.01.003</a>.

Maslach, C. (1986). Stress, burnout and workaholism. In R.R. Killberg, P.E. Nathan, y R.W. Thoreson, R.W. (Eds.), Professionals in Distress: Issues, Syndromes and Solutions in Psychology (53-73). Washington, USA: American Psychological Association.

Maslach, C., y Jackson, S. E. (1981). The measurement of experienced burnout. Journal of Organizational Behavior, 2(2), 99-113.

Maslach, C., Schaufeli, W. B., y Leiter, M. P. (2001). Job burnout. Annual Review of Psychology, 52 (1), 397-422.

Mazzetti, G., Schaufeli, W. B., y Guglielmi, D. (2014). Are workaholics born or made? Relations of workaholism with person characteristics and overwork climate. International Journal of Stress Management, 21(3), 227-254. <a href="https://doi.org/10.1037/a0035700" target="_blank"> https://doi.org/10.1037/a0035700</a>.

Mensah, J., y Amponsah-Tawiah, K. (2016). Mitigating occupational stress: the role of psychological capital. Journal of Workplace Behavioral Health, 31(4), 189-203. <a href="https://doi.org/10.1080/15555240. 2016.1198701" target="_blank"> https://doi.org/10.1080/15555240. 2016.1198701</a>.

Molino, M., Bakker, A. B., y Ghislieri, C. (2016). The role of workaholism in the job demands-resources model. Anxiety, Stress, \& Coping, $29 \quad$ (4), 400-414. <a href="https://doi.org/10.1080/10615806.2015.107083" target="_blank"> https://doi.org/10.1080/10615806.2015.107083</a>.

Moyer, F., Aziz, S., y Wuensch, K. (2017). From workaholism to burnout: psychological capital as a mediator. International Journal of Workplace Management, 10(3), 213-227. <a href="https://doi.org/10.11008/IJWHM-10-2016-0074" target="_blank"> https://doi.org/10.11008/IJWHM-10-2016-0074 </a>

Newman, A., Ucbasaran, D., Zhu, F., y Hirst, G. (2014). Psychological capital: a review and synthesis. Journal of Organizational Behavior, 35(1), 120-138. <a href="https://doi.org/10.1002/job.1916" target="_blank"> https://doi.org/10.1002/job.1916 </a>. 
$\mathrm{Ng}, \mathrm{T}$. W. H., Sorensen, K. L., y Feldman, D. C. (2007). Dimensions, antecedents, and consequences of workaholism: a conceptual integration and extension. Journal of Organizational Behavior, 28(1), 111-136. <a href="https://doi.org/10.1002/job.424" target="_blank"> https://doi.org/10.1002/job.424 $</ a>$.

Oates, W. E. (1971). Confessions of a Workaholic: The Facts about Work Addiction. Cleveland, USA: World Publishing Company.

Podsakoff, P., Mackenzie, S., Lee, J., y Podsakoff, N. (2003). Common method biases in behavioral research: a critical review of the literature and recommended remedies. Journal of Applied Psychology, 88(5), 879-903. <a href="https://doi.org/10.1037/0021-9010.88.5.879" target="_blank"> https://doi.org/10.1037/0021-9010.88.5.879</a>

Pfeffer, J. L. (1998). The human equation: building profits by putting people first. Boston, USA: Harvard Business School Press.

Salanova, M., López-González, A. A., Llorens, S., del Líbano, M., Vicente-Herrero, M. T., y Tomás-Salvá, M. (2016). Your work may be killing you! Workaholism, sleep problems and cardiovascular risk. Work \& Stress, 30(3), 228-242. <a href="https://doi.org/10.1080/02678373.2016.1203373" target="_blank"> https://doi.org/10.1080/02678373.2016.1203373</a>.

Schaufeli, W. B., Bakker, A. B., van der Heijden, F., y Prins, J. T. (2009). Workaholism, burnout, and wellbeing among junior doctors: the mediating role of role conflict. Work and Stress, 23(2), 155-172. <a href="https://doi.org/10.1080/02678370902834021"

target="_blank"> https://doi.org/10.1080/02678370902834021</a>.

Seligman, M. (1998). Learned Optimism, New York, USA: Pocket Books.

Shirom, A. (2011). Job-related burnout: a review of major research foci and challenges. In J.C. Quick, \& L.E. Tetrick (Eds.). Handbook of Occupational Health Psychology (2nd ed) (223-241). Washington, USA: American Psychological Association.

Snyder, C. R., Sympson, S., Ybasco, F., Borders, T., Babyak, M., y Higgins, R. (1996). Development and validation of the State Hope scale. Journal of Personality and Social Psychology, 70(2), 321-335. <a href="https://doi.org/10.1037/0022-3514.70.2.321" target="_blank"> https://doi.org/10.1037/0022-3514.70.2.321</a $>$.

Spence, J. T., y Robbins, A. S. (1992). Workaholism: definition, measurement, and preliminary results. Journal of Personality Assessment, $58(1), \quad 160-178 . \quad<a$ href="https://doi.org/10.1207/s15327752jpa5801_15" target="_blank"> https://doi.org/10.1207/s15327752jpa5801_15</a>.

Taris, T. W., Van Beek, I., y Schaufeli, W. B. (2012). Demographic and Occupational Correlates of Workaholism. Psychological Reports, 110, 547-554. <a href="https://doi.org/10.2466/03.09.17.PR0.110.2.547-554" target="_blank"> https://doi.org/10.2466/03.09.17.PR0.110.2.547-554</a>. 
Capital psicológico como mediador entre adicción al trabajo y burnout en trabajadoras en la industria de maquila en Guanajuato

Van Beek, I., Taris, T. W., y Schaufeli, W. B. (2011). Workaholic and work engaged employees: dead ringers or worlds apart? Journal of Occupational Health Psychology, 16(4), 468-482. <a href="https://doi.org/ 10.1037/a0024392" target="_blank"> https://doi.org/10.1037/a0024392 </a>.

Van Wijhe, C., Peeters, M., Schaufeli, W., y Hout, M. V. D. (2011). Understanding workaholism and work engagement: The role of mood and stop rules. Career Development International, 16, 254-270. <a href= "https://doi.org/10.1108/13620431111140156" target="_blank"> https://doi.org/10.1108/13620431111140156</a>.

Wang, Y., Liu, L., Wang, J., y Wang, L. (2012). Work-family conflict and burnout among Chinese doctors: the mediating role of psychological capital. Journal of Occupational Health, 54(3), 232-240. <a href="https://doi.org/10.1539/joh.11-0243-OA" target="_blank"> https://doi.org/10.1539/joh.110243-OA </a $>$.

Weigl, M., Stab, N., Herms, I., Angerer, P., Hacker, W., y Glaser, J. (2016). The associations of supervisor support and work overload with burnout and depression: a cross-sectional study in two nursing settings. Journal of Advanced Nursing, 72 (8), 1774-1788. <a href= "https://doi.org/10.1111/jan.12948" target="_blank"> https://doi.org/10.1111/jan.12948</a>. 